\title{
Pasado, presente y futuro
}

\author{
Past, present and future
}

Diana Cárdenas, MD, PhD. ${ }^{1}$

https://doi.org/10.35454/rncm.v1n1.070

En 1962 el doctor Sholom Omi Waife (1919-2011) fundador $y$, en ese entonces, editor de la revista The American Journal of Clinical Nutrition en un editorial titulado "The past, the present and the future" afirmó que:

"Esta Revista fue fundada porque se creía sinceramente que la ciencia de la nutrición estaba muy avanzada en áreas experimentales (es decir, en animales) pero muy rezagada en la aplicación clínica. También creímos, y lo hacemos ahora, que estamos en medio de un período en extremo fructífero de investigación y desarrollo en las ciencias de la nutrición. De hecho, la mitad del siglo XX bien puede ser el período de oro de esta disciplina biológica. También teníamos la esperanza de que una publicación periódica independiente, libre de presiones comerciales y con los más altos estándares, ofreciera un servicio único a las profesiones médicas y afines a traspasar las líneas tradicionales de especialización... Además, creímos que no solo The American Journal of Clinical Nutrition sería un medio de comunicación entre los científicos, sino que también debería elevar el estado de la nutrición clínica como un tema digno de mayor atención en las facultades de medicina, hospitales y, lo más importante, en la práctica clínica. Estos objetivos permanecen. Se ha comenzado, pero el objetivo aún no se ha alcanzado.” ${ }^{(1)}$.

Waife considera que el 'periodo de oro' de esta ciencia biológica está en el pasado y planteó, como prioridad, la necesidad de desarrollar la aplicación de las ciencias de la nutrición en el ámbito clínico. La comunicación es puesta en escena como una herramienta para lograr un mejor reconocimiento de la nutrición clínica. El editor resalta que partiendo de este pasado, el presente de la revista está marcado por el incremento en el número de publicaciones. Este aumento lo explica el mayor interés por la nutrición aplicada a las enfermedades humanas. Optimista frente al futuro, el editor plantea interrogantes sobre cómo responder mejor a las necesidades de los lectores. Para ello, desarrolla cuestionamientos centrales sobre el equilibrio entre las publicaciones experimentales y clínicas, sobre dónde termina la nutrición y comienzan el metabolismo, la endocrinología y la bioquímica y también acerca de la pertinencia de publicar sobre algunos temas como las dietas terapéuticas. En resumen, Waife cuestiona los límites de la nutrición clínica.

La nutrición clínica fue, es y será el objeto de la Revista de la Asociación Colombiana de Nutrición Clínica. Su pasado, presente y futuro están enmarcados en el desarrollo de esta ciencia en Colombia y en el mundo.

Pasado. Las primeras seis décadas del siglo XX permitieron el desarrollo del conocimiento en las ciencias de la nutrición. La bioquímica y el metabolismo tuvieron su auge. La Edad de Oro descrita por Waife es la antesala de lo que J.C. Stevens llamaría el "Cuarto advenimiento": la nutrición parenteral. Este advenimiento o paradigma es descrito por el doctor José Félix Patiño Restrepo (editorial invitado) en el presente número de la Revista. El Dr. Patiño, iniciador del soporte nutricional en Colombia, maestro de maestros, ha acompañado a la Asociación Colombiana de Nutrición Clínica (ACNC) desde sus inicios y hoy nos ratifica su apoyo. Gracias Dr. Patiño.

El paradigma mencionado por el Dr. Patiño se basa en una nueva concepción del nutriente: el nutriente

\footnotetext{
1 Médico Cirujano, Universidad El Bosque.

Diploma Universitario de dietética, nutrición clínica y terapéutica, Hospital Bichat, Denis Diderot, Paris, France.

Magister en Ciencias, Universidad René Descartes, Paris, France.
}

Doctorado en filosofía, Universidad de Franche-Comté, Francia. Magister en Metabolismo y Nutrición, Universidad Paris Diderot, Francia. Magister en Relaciones Internacionales, Políticas internacionales, Universidad de Lyon, Francia. 
artificial, aquel que se administra a través de una sonda o un catéter por una vía enteral o parenteral y cuya producción no es el resultado de la producción agrícola sino el resultado de la producción de la industria farmacéutica. De esta forma, la distancia entre nutriente y medicamento se acorta y con ello la posibilidad de abordar desde la clínica un problema particular: la desnutrición. El análisis de la transformación de la noción de nutriente ha hecho posible establecer, según mi investigación, desde el punto de vista epistemológico la autonomía de la nutrición clínica ${ }^{(2)}$. Aunque hoy no exista consenso internacional sobre los límites de la nutrición clínica, me atrevería a decir que sí lo hay sobre su objeto de estudio: la malnutrición (desnutrición asociada a la enfermedad, obesidad, sarcopenia, carencias de micronutrientes). Esto se traduce en un objetivo común en la práctica clínica: la lucha contra la malnutrición.

La Revista Colombiana de Metabolismo y Nutrición Clínica publicada sin interrupción dos veces por año desde 2010 con el objetivo de promover la educación y la investigación en el campo de la nutrición clínica. Lo hacía ya su predecesora, Lecturas sobre Nutrición, creada dos años después de la fundación de la ACNC en 1986. Quiero aquí resaltar el trabajo de las editoras anteriores, la enfermera Sonia Echeverri y la nutricionista Patricia Savino. Patricia fue fundadora de la ACNC y de Lecturas sobre Nutrición, y también editora de la Revista hasta el 2017. Sonia editora durante dos años, transformó Lecturas sobre Nutrición en Revista dándole un amplio enfoque interdisciplinario. Gracias a ellas y a su equipo de apoyo hoy tenemos la Revista, órgano oficial de la ACNC.

Presente. La junta directiva de la ACNC, en cabeza del Dr. Charles Bermúdez Patiño, consciente de la importancia de promover la investigación y la educación en nutrición clínica en Colombia y Latinoamérica, decide dar continuidad a la Revista. El Dr. Bermúdez me honra con el cargo de Editora y con ello el reto de su indexación en el Índice Bibliográfico Nacional Publindex de Colciencias.

Hoy, el objetivo de la Revista es seguir publicando información científica sobre el metabolismo, la terapia nutricional (nutrición enteral, suplementos orales y nutrición parenteral) y la relación entre nutrición y enfermedad. Como dije anteriormente si no hay consenso sobre los límites de la nutrición clínica como ciencia, es posible proponer una definición que se base en un análisis histórico y epistemológico y que encuentre coherencia con las guías prácticas de las principales sociedades internacionales en el campo, la Sociedad Americana de Nutrición Enteral y Parenteral (ASPEN) y la Sociedad Europea de Nutrición Clínica y Metabolismo (ESPEN).

Considero que la nutrición clínica es el resultado de una nueva visión, típica de la escuela de pensamiento del siglo XX, una visión que define la forma en que el paciente debe ser alimentado, estableciendo una estrecha relación entre desnutrición y enfermedad, y apoyado en el nuevo concepto de nutriente. En el artículo What is Clinical Nutrition?, publicado en la revista Clinical Nutrition de la ESPEN, propuse una definición de nutrición clínica basada en su estado epistemológico, su objeto y ámbito específico del conocimiento:

"La nutrición clínica es una ciencia básica interdisciplinaria y aplicada, preocupada por la malnutrición (desnutrición asociada a la enfermedad, obesidad, etc.). Su objetivo es aplicar los principios del soporte nutricional (es decir, nutrientes artificiales) en el marco de la atención o cuidado nutricional a fin de garantizar el estado nutricional y modular otras funciones biológicas para influir positivamente en el tratamiento y el resultado del paciente" (2).

Teniendo en cuenta esta definición, la ambición de internacionalizar la Revista y definir un ámbito temático particular el cual debe ser reflejado en el título sin determinantes geográficos, hemos decidido cambiar su título. La revista se estrena hoy como Revista de Nutrición Clínica y Metabolismo (RNCM).

La nutrición clínica se desarrolla en distintos espacios tanto clínicos como académicos. Los grupos de soporte nutricional creados desde finales de los años 70 luchan por seguir existiendo. La (RNCM) pretende facilitar el diálogo entre estos espacios. La nutrición clínica en Colombia, a través de la ACNC, se ha posicionado frente a sociedades científicas como FELANPE, ASPEN y ESPEN, como un país referente en la misión de alimentar a la persona enferma y lograr un adecuado cuidado nutricional hospitalario. La Revista pretende ser un canal de comunicación con estas sociedades científicas.

Lograr el objetivo de la Revista no es posible sin un equipo de trabajo y trabajo en equipo. Tanto el Comité Editorial como el Científico sufrieron una sensible renovación, congregando reconocidos académicos e investigadores nacionales e internacionales comprometidos con esta labor. Algunos de ellos han apoyado esta revista desde sus inicios. Un sincero agradecimiento para todos ellos. 
Futuro. Una nueva etapa comienza. La revista ha sido reestructurada manteniendo su propósito y alcance antes mencionados. Las secciones han sido rediseñadas para ofrecer un espacio amplio y adaptado a los temas que se desarrollan en la actualidad. Cada número tendrá artículos originales, casos clínicos, controversias y otros artículos. Se continuará dando espacio a la publicación de guías internacionales traducidas al español que consideramos importantes para la práctica del cuidado nutricional. Además, se hará énfasis en el diálogo permanente entre las ciencias básicas y la nutrición clínica, sin olvidar la epidemiología ni la salud pública. Pretendemos que la investigación básica y la investigación clínica estén al servicio de la mejora de las prácticas interdisciplinarias en nutrición clínica.

El futuro de la nutrición clínica está en su mayor y mejor difusión y en el desarrollo de la educación y la investigación. Para ello debe haber un cambio fundamental en su concepción. La nutrición clínica debe ser vista no como la aplicación de los principios y fundamentos de las ciencias de la nutrición en el ámbito clínico, sino como una ciencia autónoma, que resulta de la interacción entre medicina y nutrición humana. La nutrición clínica no debe ser más una subespecialidad clínica si no una verdadera especialidad de la medicina, enfocada desde la interdisciplinariedad. Esta concepción facilitará la asimilación e integración de nuevo conocimiento proveniente de otras disciplinas como las ciencias "omicas". Además, permitirá abordar los desafíos sociales, tecnológicos, ambientales y éticos que se aceleran en esta ciencia. Solo así entonces será: "un tema digno de mayor atención en las facultades de medicina, hospitales y, lo más importante, en la práctica clínica” ${ }^{(1)}$.

\section{Referencias bibliográficas}

1. Waife SO. Past, Present, Future. Am J Clin Nutr. 1962;10: 453-5.

2. Cárdenas D. What is Clinical Nutrition? Understanding the epistemological foundations of a new discipline. Clin. Nutr. ESPEN 2016;11:e63- e66. 